\title{
Topological Order Following a Quantum Quench
}

\author{
Dimitris I. Tsomokos ${ }^{1,2,3}$, Alioscia Hamma ${ }^{4}$, Wen Zhang ${ }^{5}$, Stephan Haas $^{5}$, Rosario Fazio ${ }^{2,6}$ \\ ${ }^{1}$ School of Physics and Astronomy, University of Hertfordshire, Hatfield AL10 9AB, UK \\ ${ }^{2}$ Scuola Normale Superiore, Piazza dei Cavalieri 7, 56126 Pisa, Italy \\ ${ }^{3}$ Department of Mathematics, Royal Holloway, University of London, Egham, TW20 0EX, UK \\ ${ }^{4}$ Perimeter Institute for Theoretical Physics, 31 Caroline St. N, N2L 2Y5, Waterloo ON, Canada \\ ${ }^{5}$ Department of Physics and Astronomy, University of Southern California, Los Angeles, CA 90089, USA \\ ${ }^{6}$ Center for Quantum Technologies, National University of Singapore, Republic of Singapore
}

(Dated: December 8, 2009)

\begin{abstract}
We determine the conditions under which topological order survives a rapid quantum quench. Specifically, we consider the case where a quantum spin system is prepared in the ground state of the Toric Code Model and, after the quench, it evolves with a Hamiltonian that does not support topological order. We provide analytical results supported by numerical evidence for a variety of quench Hamiltonians. The robustness of topological order under non-equilibrium situations is tested by studying the topological entropy and a novel dynamical measure, which makes use of the similarity between partial density matrices obtained from different topological sectors.
\end{abstract}

Quantum states with topological order (TO) belong to non-symmetry breaking phases of matter that cannot be characterized by local order parameters [1]. The absence of any local structure in these states is ultimately responsible for their robustness against certain forms of local noise. For this reason topologically ordered quantum systems with ground state degeneracy and persistent energy gap, such as the Toric Code Model (TCM), have been proposed as robust quantum memories [2]. Moreover, systems with TO can support non-Abelian anyons as quasiparticle excitations, in which case universal quantum computation based on topological, and thus more robust, interactions is indeed possible [3].

Evaluating the persistence of topological order under adverse conditions is a crucial step towards future experimental realizations of topological quantum hardware. The robustness of the TCM has recently been studied with respect to noise [4] and thermal equilibrium at a finite temperature $5,6,6,7]$. The quantum phase transition from topological to magnetic order in the TCM has also been analyzed 8, 9, 10]. We can say that a system possesses TO if it is gapped and it has a degenerate ground state whose degeneracy depends on the system topology. If topology is to be responsible for the degeneracy, it is expected that different ground states should be indistinguishable locally. We show here that this is certainly the case for the TCM and use this property to characterize the presence of TO. In the absence of a local order parameter, establishing the presence of $\mathrm{TO}$ is a daunting task. An effective order parameter for TO is the topological entanglement entropy [11, 12], $S_{\text {topo: }}$ if $S_{\text {topo }}(|\psi\rangle)=0$, the state $|\psi\rangle$ does not have TO; while if $S_{\text {topo }}(|\psi\rangle)>0$, the state has TO. $S_{\text {topo }}$ has already given insights into the nature of the TCM and its transitions to non-topological phases [6, 7, [8, 9]. By contrast, the dynamical properties of TO have remained largely unexplored.

In this work we investigate the dynamical response of a state with TO to a sudden quench, a topic that has attracted a lot of attention recently both experimentally
[13] and theoretically [14]. If the dynamics of the system is governed by a Hamiltonian that does not support TO then it is crucial to determine the extent to which TO survives and to assess the nature of destructive perturbations. In particular, we examine the case of a fast quench starting from the ground state of the TCM [15]. We determine the influence of such a quench on the TO by studying the dynamical behaviour of the block entanglement entropy [16] and the topological entanglement entropy, whose dynamics is calculated here for the first time. In addition we introduce a new dynamical measure of TO, which is based on the similarity between states obtained from different topological sectors.

We start by considering a system of spin- $\frac{1}{2}$ particles situated on the edges of a square lattice with periodic boundary conditions. The TCM Hamiltonian on such a lattice is $H_{0}=-\sum_{s} A_{s}-\sum_{p} B_{p}$, where $A_{s}=\otimes_{l \in s} X_{l}$ is a star operator applied to spins on edges that meet on a vertex and $B_{p}=\otimes_{l \in p} Z_{l}$ is a plaquette operator on edges that form a square ( $X$ and $Z$ denote the usual Pauli operators). These operators stabilize the ground state of the model, that is, $\left|\Psi_{0}\right\rangle=A_{s}\left|\Psi_{0}\right\rangle=B_{p}\left|\Psi_{0}\right\rangle$. There is fourfold degeneracy in the ground state corresponding to different topological sectors [2]. This is because the incontractible strings along the vertical and horizontal directions, denoted by $w_{1}$ and $w_{2}$, commute with the Hamiltonian and map $\left|\Psi_{0}\right\rangle$ into orthogonal sectors. The ground state manifold can thus be written as $\mathcal{L}=\operatorname{span}\left\{w_{1}^{i} w_{2}^{j}\left|\Psi_{0}\right\rangle ; i, j=0,1\right\}$ with $\left|\Psi_{0}\right\rangle=$ $|G|^{-1 / 2} \sum_{g \in G} g|\mathbf{0}\rangle$, where $g$ is a string of star operators and $|\mathbf{0}\rangle \equiv|00 \cdots 0\rangle$ is a reference state (vacuum) in which all the spins are pointing in the $+Z$ direction (equivalently, one may choose to define the vacuum in the $X$ basis and the strings $g$ in the $Z$-basis). The Abelian group $G$ of all star operators on an $m \times n$ lattice with $N=2 m n$ spins is generated under tensor multiplication. Due to the periodic boundary conditions, $|G|=2^{m n-1}$.

The lattice is prepared in the ground state $\left|\Psi_{0}\right\rangle$ of the TCM Hamiltonian $H_{0}$. At $t=0$ a different Hamiltonian $H$ is suddenly switched on. The evolution is then given 
by $|\Psi(t)\rangle=\exp (-i H t)\left|\Psi_{0}\right\rangle$. If $\left\{\epsilon_{l},\left|\phi_{l}\right\rangle\right\}$ is the spectrum of $H$ then $\exp (-i H t)=\sum_{l=1}^{2^{N}} \exp \left(-i \epsilon_{l} t\right)\left|\phi_{l}\right\rangle\left\langle\phi_{l}\right|$ and so

$$
|\Psi(t)\rangle=\frac{1}{\sqrt{|G|}} \sum_{l=1}^{2^{N}} \sum_{g \in G}\left\langle\phi_{l}|g| \mathbf{0}\right\rangle \exp \left(-i \epsilon_{l} t\right)\left|\phi_{l}\right\rangle .
$$

This is the most general form of the quenched state.

Firstly we prove that there exist families of quench Hamiltonians that cannot disturb TO. To this end we consider that the eigenstates of $H$ can be written as general spin flips $x$ acting on the vacuum, i.e., $\left|\phi_{l}\right\rangle=x|\mathbf{0}\rangle$. The spin flips define a group $\mathcal{X}$ under tensor multiplication, $\left\{x \in \mathcal{X} \mid x=X_{i} \otimes X_{j} \otimes \cdots\right\}$. To apply the argument also to the group $\mathcal{Z}$ of all $Z_{l}$-operators, we take the initial state to be in a general superposition of the four topological sectors. Then the quenched state of Eq. (1) becomes $|\psi(t)\rangle=|G|^{-1 / 2} \sum_{g \in G, x_{l} \in \mathcal{X}, i, j} \alpha_{i j} \exp \left[-i \epsilon_{l} t\right] F_{i j}\left|\phi_{l}\right\rangle$ with $F_{i j} \equiv\left\langle\mathbf{0}\left|x_{l} w_{1}^{i} w_{2}^{j} g\right| \mathbf{0}\right\rangle$. The only surviving terms are those such that $x_{l}=w_{1}^{i} w_{2}^{j} g \equiv g^{i j}$ and therefore we obtain

$$
|\psi(t)\rangle=\frac{1}{\sqrt{|G|}} \sum_{g \in G, i j=0,1} \alpha_{i j} \exp \left[-i \epsilon\left(g^{i j}\right) t\right] g^{i j}|\mathbf{0}\rangle .
$$

The entanglement between a contractible region $A$ of the lattice and its complement, $B$, is given by the entanglement entropy $S_{A}=-\operatorname{Tr}\left(\rho_{A} \ln \rho_{A}\right)$, where $\rho_{A}$ is the reduced state corresponding to region $A$. Using the replica trick [17], $S_{A}(t)=-\lim _{n \rightarrow 1} \partial_{n} \operatorname{Tr}\left[\rho_{A}^{n}(t)\right]$, where $\rho_{A}(t)=$ $\operatorname{Tr}_{B}[|\Psi(t)\rangle\langle\Psi(t)|]$ is calculated for the general quenched state. Specifically for the state of Eq. (2), we have $\rho(t)=|G|^{-1} \sum_{g, \tilde{g} \in G, i j k l=0,1} \alpha_{i j} \alpha_{k l}^{*} \mathcal{E}^{i j k l}(g, g \tilde{g}) g|\mathbf{0}\rangle\langle\mathbf{0}| g \tilde{g}$, where $\mathcal{E}^{i j k l}(g, \bar{g}) \equiv \exp \left[-i \epsilon\left(g^{i j}\right) t\right] \exp \left[i \epsilon\left(\bar{g}^{k l}\right) t\right]$ and we have used the property $\bar{g}=g \tilde{g}$ of the group $G$. In order to calculate the state $\rho_{A}(t)=\operatorname{Tr}_{B}[\rho(t)]$ we distinguish the region, $|\mathbf{0}\rangle \equiv\left|0_{A}\right\rangle \otimes\left|0_{B}\right\rangle$, and we also define the subgroups of $G$ that act trivially on the subsystems, i.e., $G_{A} \equiv\left\{g \in G \mid g=g_{A} \otimes \mathbb{1}_{B}\right\}$ and $G_{B} \equiv\left\{g \in G \mid g=\mathbb{1}_{A} \otimes g_{B}\right\}$. Since $A$ is contractible, there are always group elements $h_{1}, h_{2} \in G$ such that $h_{i} w_{i} \in G_{B}$ for $i=1,2$. In other words, it is always possible to deform the loop $w_{i}$ so as to move it out of the region $A$. Therefore, we have $\rho_{A}(t)=$ $|G|^{-1} \sum_{g \in G, \tilde{g} \in G_{A}, i j=0,1}\left|\alpha_{i j}\right|^{2} \mathcal{E}^{i j i j}(g, g \tilde{g}) g_{A}\left|0_{A}\right\rangle\left\langle 0_{A}\right| g_{A} \tilde{g}_{A}$. Notice that for the ground state of the TCM, $\rho_{A}$ does not depend on the coefficients $\alpha_{i j}$, meaning that two orthogonal ground states are locally identical.

The particular structure of the Hamiltonian $H$ implies that $\epsilon\left(g^{i j}\right)=k^{i j} \epsilon(g)$ where $k^{i j}$ is a constant; hence $\rho_{A}^{n}(t)=|G|^{-n} \sum_{g_{l} \in G, \tilde{g}_{l} \in G_{A}} \Pi(t) \quad g_{1, A}\left|0_{A}\right\rangle\left\langle 0_{A}\right| g_{1, A} \tilde{g}_{1, A}$ $g_{2, A}\left|0_{A}\right\rangle\left\langle 0_{A}\left|g_{2, A} \tilde{g}_{2, A} \cdots g_{n, A}\right| 0_{A}\right\rangle\left\langle 0_{A}\right| g_{n, A} \tilde{g}_{n, A}$, where the $\Pi(t) \equiv \mathcal{E}\left(g_{1}, g_{1} \tilde{g}_{1}\right) \mathcal{E}\left(g_{2}, g_{2} \tilde{g}_{2}\right) \cdots \mathcal{E}\left(g_{n}, g_{n} \tilde{g}_{n}\right)$ includes the time dependence of the state. Each expectation value of the form $\left\langle 0_{A}\left|g_{l, A} \tilde{g}_{l, A} g_{l+1, A}\right| 0_{A}\right\rangle$ is set equal to 1 and this imposes the condition $g_{l+1, A}=g_{l, A} \tilde{g}_{l, A}$ and so $g_{l} \tilde{g}_{l} g_{l+1} \in G_{B}$. In this way we get $\rho_{A}^{n}(t)=\left|G_{B}\right|^{n}|G|^{-n}$ $\sum_{g_{1, n} \in G, \tilde{g}_{n} \in G_{A}} \Pi(t) \quad g_{1, A}\left|0_{A}\right\rangle\left\langle 0_{A}\right| g_{n, A} \tilde{g}_{n, A}$, where now $\Pi(t)=\mathcal{E}\left(g_{1}, g_{n} \tilde{g}_{n}\right)$. Using the replica trick we find
$S_{A}(t)=\ln \left(|G| /\left|G_{A}\right|\left|G_{B}\right|\right)$. Therefore the entanglement entropy of the quenched state $|\psi(t)\rangle$ of Eq. (2) remains constant in time. Moreover this state obeys an area law for the entanglement, $S=L_{A}-1$, where $L_{A}$ is the length of the boundary of $A$ and the universal finite correction signals the presence of TO [12].

For the topological entropy we use the expression due to Levin and Wen [11], $S_{\text {topo }}=-S_{A B C D}+S_{A B C}+$ $S_{A C D}-S_{A C}$ where $A, B, C, D$ are the sides of a $2 \times 2$ square in the lattice each containing two spins [8]. Evidently, $S_{\text {topo }}$ is time-independent for the quenched state of Eq. (2) since the block entanglement entropy is constant for this state. This completes the proof of the first result, which can be stated as follows: $S_{\text {topo }}(t)=1$ if the eigenstates of the quench Hamiltonian can be written as spin flips on the vacuum (in either basis). For this reason we call such quenches non-destructive. Any Hamiltonian $H(Z)$ or $H(X)$ that depends on only one Pauli operator has such eigenstates. Physically relevant cases include $H_{1}=-\sum_{i=1}^{N} h_{i} S_{i}$ and $H_{2}=-\sum_{\langle i, j\rangle} J_{i} S_{i} S_{j}$, where $S=X, Z$ and $i, j$ are nearest neighbors. In the absence of disorder (i.e., for $h_{i}=h$ and $J_{i}=J$ ) we have $\left|\left\langle\Psi_{0} \mid \psi(t)\right\rangle\right|$ $=|G|^{-1}\left|\sum_{g \in G} \cos [E(g) t]\right|$, where $E(g)$ are the eigenenergies in either case. Using the commutativity of terms in $H_{1,2}$ it is easy to show that such quenches have a period of recurrence $T=\pi / 2 h, \pi / 2 J$ independently of the lattice size. For this reason, we say that such a quench is trivial. Moreover, the entanglement remains constant at all times even in the disordered case. Conversely, a quench will be non-trivial if the periodicity is lost in the large system limit and the recurrence time increases with the system size. In order to detect the periodicity of the system we will study the overlap $\left|\left\langle\Psi_{0} \mid \Psi(t)\right\rangle\right|$ between the initial state $\left|\Psi_{0}\right\rangle$ and the quenched state of Eq. (1).

In order to detect how TO is preserved by a non-trivial quench we use $S_{\text {topo }}$ and, in addition, the Uhlmann fidelity $\mathcal{F}(t)$ between reduced systems that correspond to orthogonal ground states. The $\mathcal{F}(t)$ is given by $\mathcal{F}(t)=\operatorname{Tr} \sqrt{\sqrt{\sigma_{A}^{i j}(t)} \sigma_{A}^{k l}(t) \sqrt{\rho_{A}^{i j}(t)}}$, where $\sigma^{i j}(t=0)$ is the density matrix of $w_{1}^{i} w_{2}^{j}\left|\Psi_{0}\right\rangle$. As we have seen above, in the topological phase the reduced systems are indistinguishable and $\mathcal{F}(0)=1$, by construction. In numerical calculations with finite size systems we need to choose the region $A$ such that its bulk contains more degrees of freedom than the boundary. For this reason we use this measure only for maximum available lattice sizes.

We are now ready to examine non-trivial quench Hamiltonians for small lattices by means of exact numerical diagonalization. The quantities of interest are $\left|\left\langle\Psi_{0} \mid \Psi(t)\right\rangle\right|, S_{\text {topo }}(t)$ and $\mathcal{F}(t)$. We start with

$$
H_{3}=-J_{1} \sum_{i} Z_{i}-J_{2} \sum_{\langle i, j\rangle} X_{i} X_{j}
$$

For $J_{1} \gg J_{2}$ this Hamiltonian reduces to $H_{1}$, while for $J_{2} \gg J_{1}$ it reduces to $H_{2}$. We have shown that for both of these extremes the TO of the initial state is robust 


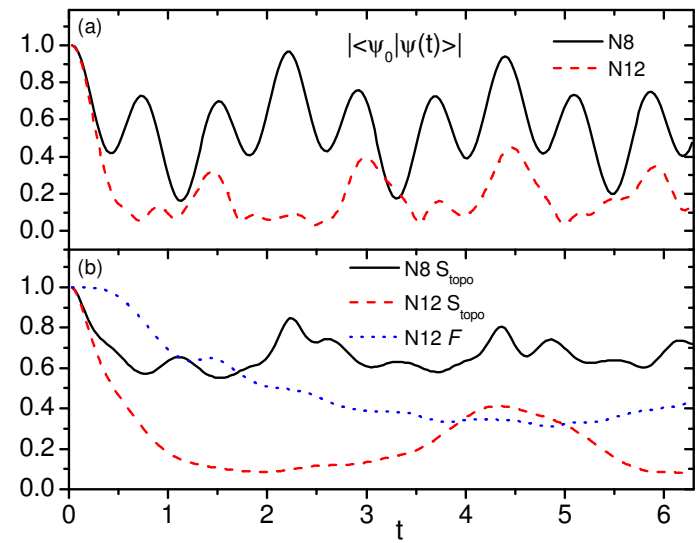

FIG. 1: (Color online) Numerical results for the quench $\mathrm{H}_{3}$ with couplings $J_{1} / J_{2}=0.33$. (a) Absolute overlap $\left|\left\langle\Psi_{0} \mid \Psi(t)\right\rangle\right|$ on lattices with $N=8,12$ spins. (b) $S_{\text {topo }}(t)$ for the $N=8,12$ lattices and fidelity $\mathcal{F}(t)$ for the $N=12$ lattice.

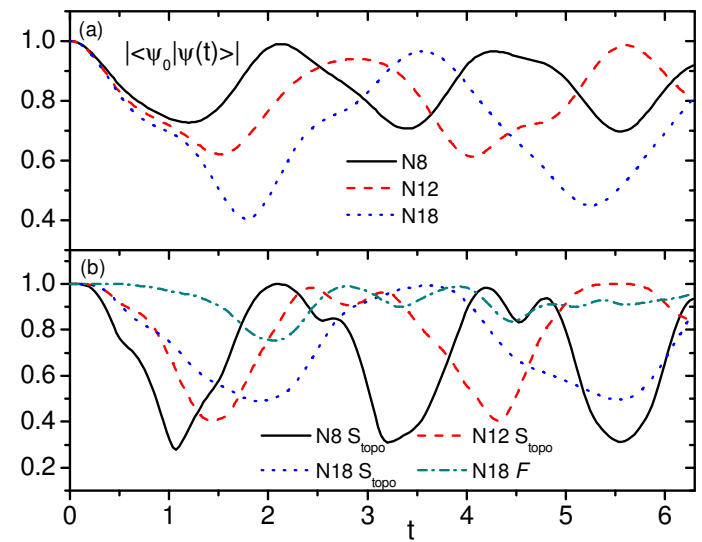

FIG. 2: (Color online) Numerical results for the quench $H_{4}$ with $h=0.34$. (a) Absolute overlap $\left|\left\langle\Psi_{0} \mid \Psi(t)\right\rangle\right|$ on lattices with $N=8,12,18$ spins. (b) $S_{\text {topo }}(t)$ for the $N=8,12,18$ lattices and fidelity $\mathcal{F}(t)$ for the $N=18$ lattice.

during the evolution. By contrast, in the intermediate regime, the recurrence time increases with the size of the system. The two competing terms affect both loop structures that support TO. The $S_{\text {topo }}$ and the $\mathcal{F}(t)$ quickly drop to low values (we note that $\mathcal{F}(t)$ is typically more sensitive numerically than $S_{\text {topo }}$ ). The results are presented in Fig. 1. The system undergoes an eigenstate thermalization in which TO is destroyed. Having used several values of $J_{1} / J_{2}$ we find that the most disrupting case for TO occurs for $J_{1} / J_{2} \approx 0.33$, which is the precursor of the quantum critical point for $\mathrm{H}_{3}$. In Ising-like models the speed of interactions is $\sim \sqrt{J_{1} J_{2}}$ [18] and is maximum at the critical point. At this value correlations spread faster in the lattice and the system samples a greater number of states in the Hilbert space.

Are there non-trivial quenches that preserve topological order? At first sight this would seem unlikely because in such cases the total state typically explores large parts

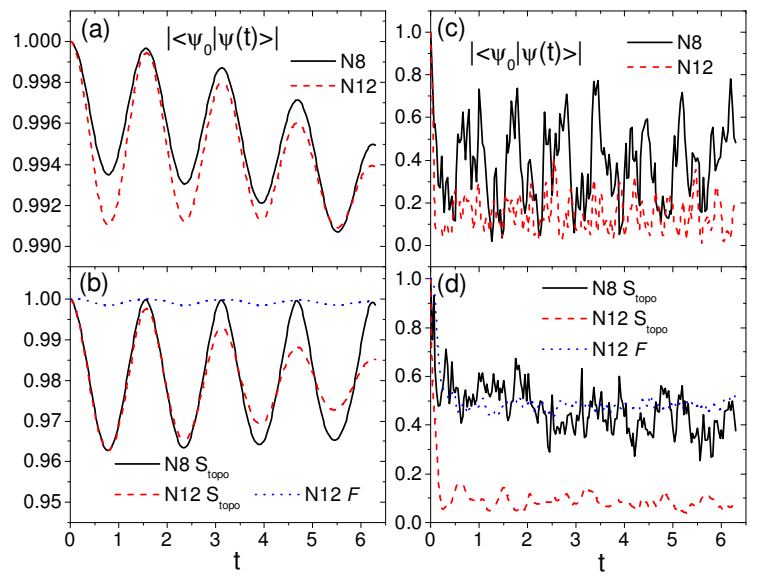

FIG. 3: (Color online) Numerical results for the quench $H_{5}$. (a) $\left|\left\langle\Psi_{0} \mid \Psi(t)\right\rangle\right|$ on the $N=8,12$ lattices for $J_{1}=J_{2}=0.033$. (b) $S_{\text {topo }}(t)$ for the $N=8,12$ lattices and $\mathcal{F}(t)$ for the $N=12$ lattice for $J_{1}=J_{2}=0.033$. (c) The same as in (a) but for $J_{1}=J_{2}=3.3$. (d) The same as in (b) but for $J_{1}=J_{2}=3.3$

of the Hilbert space. However the Hamiltonian

$$
H_{4}=-\sum_{s} A_{s}-\sum_{p} B_{p}-h \sum_{i} Z_{i}
$$

displays interesting behavior. A reasonable scenario is one where the system is prepared in the ground state of $H_{4}$ for $h=0$ and then it is let to evolve for some $h \neq 0$. This can happen, e.g., if the ground state of the TCM is precisely prepared using syndrome measurements but there is some defect in the laboratory implementation. The model was analyzed in Ref. [8] and it was shown that there is a precursor of a quantum phase transition even for small systems (for $N=8$ we have $h_{c} \approx 0.34$ ). In particular, it was shown there that for $h<h_{\mathrm{c}}$ the system is TO and for $h>h_{\mathrm{c}}$ it is normally ordered.

We find that this quench is non-trivial: as the system size increases the overlap $\left|\left\langle\Psi_{0} \mid \Psi(t)\right\rangle\right|$ becomes smaller and recurrences occur later. In Fig. 2 we present the results for $h=0.34$. The influence of this quench on the system is milder than $H_{3}$ due to the fact that $H_{4}$ couples the initial state to a much smaller Hilbert space as it is constrained by $B_{p}=1$ for every plaquette $p$. On the other hand, the time-averaged $S_{\text {topo }}$ increases with the system size. For small values of $h$ this is in line with the statical behavior analyzed in Ref. [8]: the ground state of $H_{4}$ for $h \ll 1$ has TO and $S_{\text {topo }}$ approaches the maximum value in the thermodynamic limit. Nevertheless, there is an important difference with the statical case in that large values of $h$ destroy TO (the ground state of $H_{4}$ for $h \gg 1$ is a trivial separable state). For the sudden quench, by contrast, large values of $h$ preserve TO as the size of the lattice increases.

We also present a non-trivial quench where the different possible phases co-exist in the dynamical behavior,

$$
H_{5}=-\sum_{s} A_{s}-\sum_{p} B_{p}-J_{1} \sum_{i} Z_{i}-J_{2} \sum_{\langle i, j\rangle} X_{i} X_{j} .
$$


For $J_{1} \gg J_{2}>0.33$ or $J_{2} \gg J_{1}>0.34$ the system undergoes a periodic dynamic as with the quenches $H_{1}, H_{2}$. If $J_{2} \ll J_{1} \sim 0.34$ and $J_{1} \ll J_{2} \sim 0.33$ the system has a non-trivial dynamic behavior where TO is preserved as with $H_{4}$. Finally we have the most interesting case when $J_{1} \sim J_{2}$ (see Fig. 3). Here we have two different phases: if $J_{1} \gg 0.33$, the quench is destructive; but if $J_{1} \ll 0.33$, TO is preserved, which has been verified by checking that the time-averaged topological entropy increases with lattice size.

In summary, we investigated how a topologically ordered ground state reacts to rapid quenches. TO was detected by the topological entropy and a new dynamical quantity, the fidelity between reduced states belonging to different topological sectors. Clearly TO is preserved if the quench Hamiltonian has a ground state belonging to a topologically ordered phase. Nevertheless, we showed that quenches in a different phase can still preserve TO. On the other hand, we also showed that there is a class of quench Hamiltonian (see Eq.(3)), whose dy- namics leads to a suppression of TO. The destructive dynamics results from Hamiltonians that are both nontopologically ordered and have a finite propagation of interactions through the lattice. It is remarkable that once a state with TO has been initialized, it can be more robust with respect to dynamical evolution, as opposed to statical perturbations. It would be interesting to study the evolution of the TCM in the presence of a suitable noise model under various quenches. This would be a first step in constructing a dynamical decoupling protocol [19] for the protection of the TCM from thermal noise.

We thank P. Calabrese, J. Pachos, P. Zanardi for useful comments. We acknowledge financial support by the EPSRC (D.I.T., grant no. EP/D065305/1), the National Research Foundation and Ministry of Education Singapore (R.F.), the NSF (S.H., grant no. DMR-0804914) and the Perimeter Institute for Theoretical Physics. We also thank the USC Center for High Performance Computing and Communications for use of facilities.
[1] X.-G. Wen, Quantum Field Theory of Many-Body Systems (Oxford University Press, 2004).

[2] A. Kitaev, Ann. Phys. 303, 2 (2003); E. Dennis, A. Kitaev, A. Landahl, and J. Preskill, J. Math. Phys. 43, 4452 (2002).

[3] C. Nayak, S. H. Simon, A. Stern, M. Freedman, S. Das Sarma, Rev. Mod. Phys. 80, 1083 (2008).

[4] S. Trebst, P. Werner, M. Troyer, K. Shtengel, C. Nayak, Phys. Rev. Lett. 98, 070602 (2007).

[5] R. Alicki, M. Fannes, M. Horodecki, J. Phys. A: Math. Theor. 40, 6451 (2007); Z. Nussinov and G. Ortiz, Phys. Rev. B 77, 064302 (2008); A. Kay, Phys. Rev. Lett 102, 070503 (2009).

[6] C. Castelnovo and C. Chamon, Phys. Rev. B 76, 184442 (2007).

[7] S. Iblisdir, D. Perez-Garcia, M. Aguado, J. Pachos, Phys. Rev. B 79134303 (2009).

[8] A. Hamma, W. Zhang, S. Haas, D. A. Lidar, Phys. Rev. B 77, 155111 (2008).

[9] C. Castelnovo and C. Chamon, Phys. Rev. B 77, 054433 (2008).

[10] J. Vidal, S. Dusuel, K. P. Schmidt, Phys. Rev. B 79, 033109 (2009); I. S. Tupitsyn, et al., arXiv:0804.3175

[11] A. Kitaev and J. Preskill, Phys. Rev. Lett. 96, 110404 (2006); M. Levin and X.-G. Wen, ibid. 96, 110405 (2006).

[12] A. Hamma, R. Ionicioiu, P. Zanardi, Phys. Rev. A 71, 022315 (2005).

[13] M. Greiner, O. Mandel, T. Esslinger, T. W. Hänsch, I. Bloch, Nature 415, 39 (2002); T. Kinoshita, T. Wenger, D. S. Weiss, Nature 440, 900 (2006); T. Stöferle, H. Moritz, C. Schori, M. Köhl, T. Esslinger, Phys. Rev. Lett. 92, 130403 (2004); C. D. Fertig, K. M. O'Hara, J. H. Huckans, S. L. Rolston, W. D. Phillips, J. V. Porto, Phys. Rev. Lett. 94, 120403 (2005).

[14] F. Iglói and H. Rieger, Phys. Rev. Lett. 85, 3233
(2000); E. Altman and A. Auerbach, Phys. Rev. Lett. 89, 250404 (2002); K. Sengupta, S. Powell, S. Sachdev, Phys. Rev. A 69, 053616 (2004); P. Calabrese and J. Cardy, Phys. Rev. Lett. 96, 136801 (2006); M. Rigol, A. Muramatsu, M. Olshanii, Phys. Rev. A 74, 053616 (2006); C. Kollath, A. M. Läuchli, E. Altman, Phys. Rev. Lett. 98, 180601 (2007); V. Gritsev, E. Demler, M. Lukin, A. Polkovnikov, Phys. Rev. Lett. 99, 200404 (2007). M. Cramer, C. M. Dawson, J. Eisert, T. J. Osborne, Phys. Rev. Lett. 100, 030602 (2008); T. Barthel and U. Schollwock, Phys. Rev. Lett. 100, 100601 (2008); A. Silva, Phys. Rev. Lett. 101, 120603 (2008), L. Campos Venuti and P. Zanardi, arXiv:0907.0683.

[15] The opposite situation of slow (but non-adiabatic) dynamics in the honeycomb lattice model was studied by K. Sengupta, D. Sen, S. Mondal, Phys. Rev. Lett. 100, 077204 (2008); S. Mondal, D. Sen, K. Sengupta, Phys. Rev. B 78, 045101 (2008). It was shown that the density of defects shows peculiar signatures of the topological phase transition.

[16] The dynamics of the block entropy has been studied by a variety of methods after the initial work of Calabrese and Cardy [17]. For a review, see also L. Amico, R. Fazio, A. Osterloh, V. Vedral, Rev. Mod. Phys. 80, 517 (2008).

[17] P. Calabrese and J. Cardy, J. Stat. Mech.: Theory Exp. P04010 (2005); G. De Chiara, S. Montangero, P. Calabrese, R. Fazio, J. Stat. Mech.: Theory Exp. P03001 (2006); J. Eisert and T. J. Osborne, Phys. Rev. Lett. 97, 150404 (2006); P. Calabrese and J. Cardy, J. Stat. Mech.: Theory Exp. P10004 (2007).

[18] A. Hamma, F. Markopoulou, I. Premont-Schwarz, S. Severini, Phys. Rev. Lett. 102, 017204 (2009).

[19] K. Khodjasteh and D. A. Lidar, Phys. Rev. Lett. 95, 180501 (2005). 